\title{
Buckling mosaic of a circular plate on a partial elastic foundation
}

\author{
L.H. Yu \\ Department of Mathematics, National Chung Cheng University, Min-Hsiung, Chia-Yi 621, Taiwan, ROC \\ C.Y. Wang \\ Departments of Mathematics and Mechanical Engineering, Michigan State University \\ East Lansing, MI 48824, USA \\ (Received January 22, 2009, Accepted August 14, 2009)
}

Keywords: buckling; plate; elastic foundation.

\section{Introduction}

Buckling of plates resting on an elastic foundation is important in numerous practical applications such as concrete pavements and foundations on soil. The buckling of circular plates has been reported by several authors (Yu 1957, Galletly 1959, Kline and Hancock 1965, Wolkowisky 1969, Gupta et al. 1991, Gupta and Ansari 1998, Wang 2005, Gupta et al. 2006). All of the above sources considered a circular plate resting on a full elastic foundation. The present Note studies the buckling of a circular plate on a partial concentric elastic foundation which is important for centrally supported or stiffened plates. We shall investigate whether there are any other asymmetric buckling modes aside from those reported by Wang (2005).

Our solutions will also be exact, such that no numerical errors are incurred. Exact buckling criteria also serve as standards for checking results from approximate or numerical methods (Wang et al. 2005).

\section{Formulation}

Consider a thin (Kirchkoff) elastic plate of radius $R$ centrally supported on a partial linear (Winkler) foundation of radius $c R$. The outer edge of the plate is compressed by a uniform load $N$, and may be clamped, simply-supported, or free. Let $w_{1}$ be the lateral deflection of the plate in the region not supported by the foundation and $w_{2}$ be that supported by the foundation. The governing

† Corresponding author, E-mail: cywang@mth.msu.edu 
equations are

$$
\begin{gathered}
\nabla^{4} w_{1}+\lambda^{2} \nabla^{2} w_{1}=0, \quad 1 \geq r>c \\
\nabla^{4} w_{2}+\lambda^{2} \nabla^{2} w_{2}+\gamma^{4} w_{2}=0, \quad c>r \geq 0
\end{gathered}
$$

Here $\lambda^{2}=N R^{2} / D, \gamma^{4}=k R^{4} / D, D$ being the flexural rigidity of the plate, $k$ the spring constant of the foundation, and lengths are normalized with $R$. Let $w_{j}=u_{j}(r) \cos (n \theta)$ where $n$ is the number of nodal diameters. The general solution to Eq. (1) is

$$
u_{1}=a_{1} J_{n}(\lambda r)+a_{2} Y_{n}(\lambda r)+a_{3} r^{n}+a_{4} r^{-n}
$$

except for $n=0$, the last term is replaced by $a_{4} \ln r$. For the supported region, the general solution depends on the foundation stiffness. If $\lambda>\sqrt{2} \gamma$

$$
u_{2}=a_{5} J_{n}(\alpha r)+a_{6} J_{n}(\beta r)
$$

where $\alpha=\sqrt{\left(\lambda^{2}+\sqrt{\left.\lambda^{4}-4 \gamma^{4}\right) / 2}\right)}, \beta=\sqrt{\left(\lambda^{2}-\sqrt{\left.\lambda^{4}-4 \gamma^{4}\right) / 2}\right.}$.

$$
\text { If } \lambda=\sqrt{2} \gamma
$$

$$
u_{2}=a_{5} J_{n}(\gamma r)+a_{6} r J_{n+1}(\gamma r)
$$

If $\lambda<\sqrt{2} \gamma$

$$
u_{2}=a_{5} \operatorname{Re}\left[J_{n}(i \delta r)\right]+a_{6} \operatorname{Im}\left[J_{n}(i \delta r)\right]
$$

where $i=\sqrt{-1}$ and $\delta=\sqrt{\left(-\lambda^{2}+\sqrt{4 \gamma^{4}-\lambda^{4} i}\right) / 2}$. At $r=c$, continuity in displacement, slope, moment and shear are equivalent to

$$
\begin{array}{cc}
u_{1}(c)=u_{2}(c), & u_{1}^{\prime}(c)=u_{2}^{\prime}(c) \\
u_{1}^{\prime \prime}(c)=u_{2}^{\prime \prime}(c), & u_{1}^{\prime \prime \prime}(c)=u_{2}^{\prime \prime \prime}(c)
\end{array}
$$

where the prime represents a derivative with respect to $r$. There are two more standard conditions at the outer edge depending on whether it is clamped, simply supported or free. For non-trivial solutions, the six conditions yield a characteristic equation. The lowest $\lambda$ gives the buckling force $N$. A routine root finder can generate the eigenvalues to any accuracy.

\section{Results}

For the clamped case (Fig. 1) the buckling load rises monotonically with both $\lambda$ and $c$. When the foundation is absent, or $c=0$, the buckling load is the first root of $J_{1}(\lambda)=0$ or $\lambda=3.8317$. For full foundation, or $c=1$, our solution agrees with those of Wang (2005). However, there is a mosaic of mode switches for partial foundations. We see that there are regions of higher modes, from $n=0$ to $n=4$ nodal diameters in the range studied. In general, for fixed partial foundations the buckling starts with an axisymmetric mode $(n=0)$ for small foundation stiffness, then the number of nodal diameters increases as $\gamma$ is increased. The phenomenon is also present for beams on elastic foundation (Hetenyi 1948). 


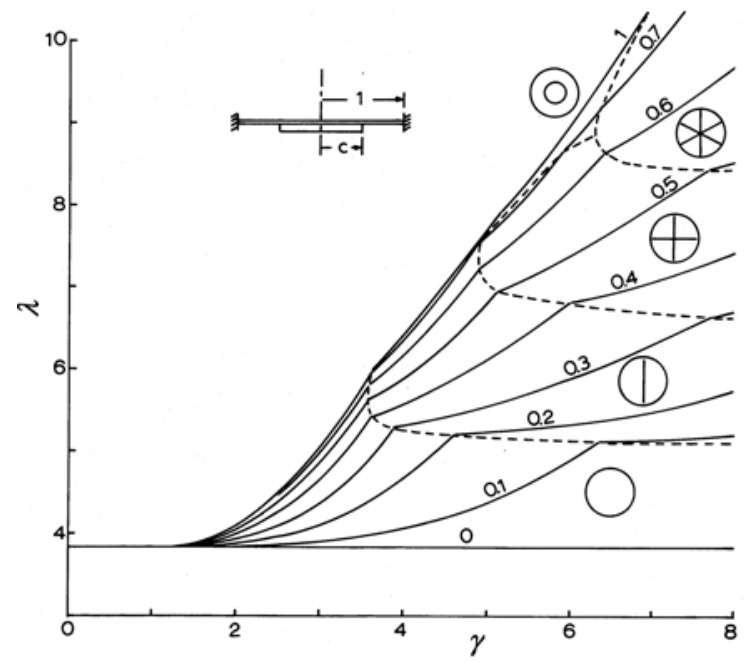

Fig. 1 The clamped case. The buckling load $\lambda$ versus foundation stiffness $\gamma$. Dashed lines denote mode changes. Numerals denote the foundation size $c$. Typical modes are depicted

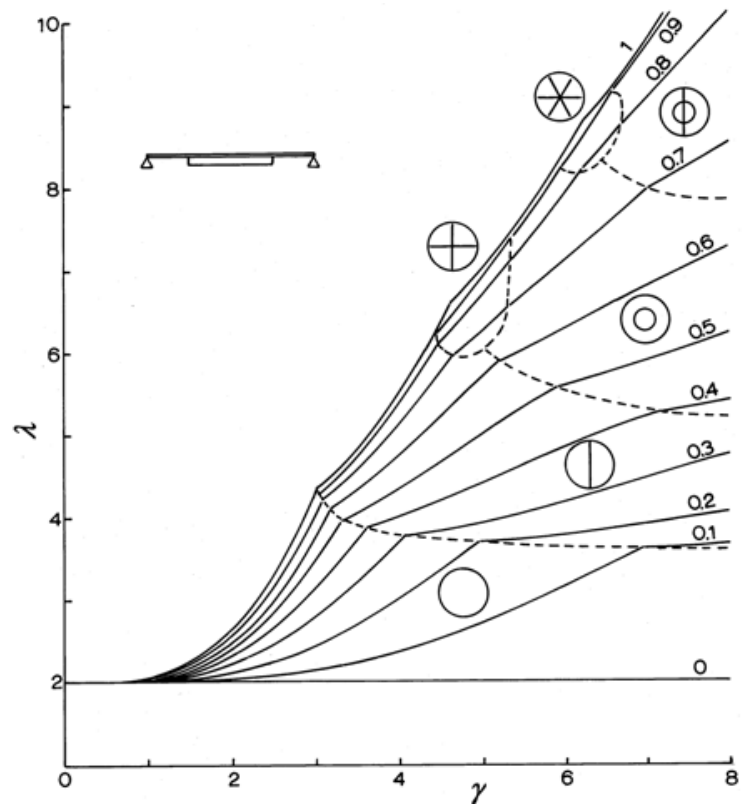

Fig. 2 The simply supported case. Legend same as Fig. 1

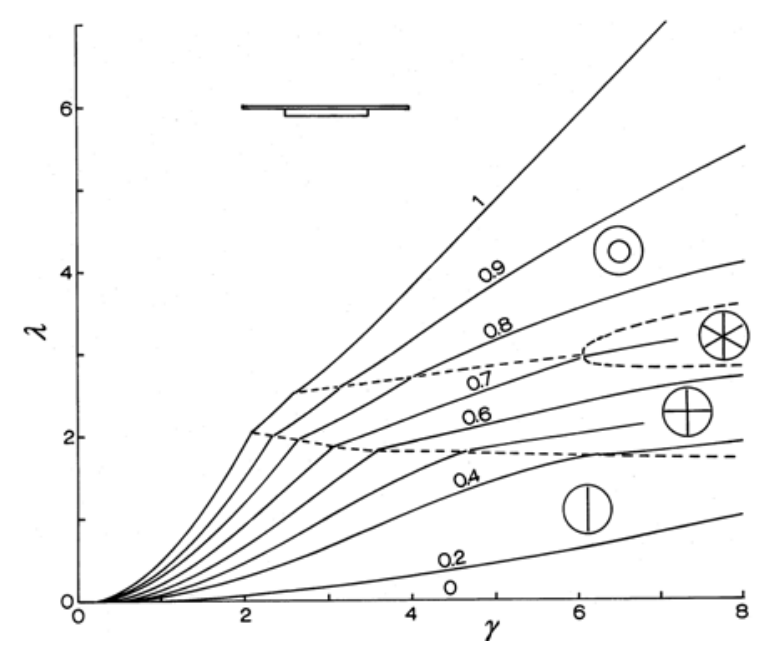

Fig. 3 The free edge case. Legend same as Fig. 1

Fig. 2 shows the simply supported case. When the foundation is absent, the buckling load is 2.0489 , or the first root of $\lambda J_{0}(\lambda)-(1-v) J_{1}(\lambda)=0$, where $v$ is taken as 0.3 . We see there are large pockets of higher modes $(n=2,3)$ for larger $c$. For fixed moderate $c$, the buckling modes tend to switch between zero and one nodal diameters in the range studied. This does not preclude the appearance of higher modes when the stiffness becomes much larger.

Fig. 3 shows the free edge case. All curves now start with zero buckling load, representing a rigid 
Table 1 The buckling load for various foundation size and edge conditions. Number in parenthesis denote the number of nodal diameters.

\begin{tabular}{llcccc}
\hline \hline & Edge & $\gamma=2$ & $\gamma=4$ & $\gamma=6$ & $\gamma=8$ \\
\hline \multirow{3}{*}{$c \approx 0$} & Clamped & $3.832(0)$ & $3.832(0)$ & $3.832(0)$ & $3.832(0)$ \\
& Sim-Supp & $2.049(0)$ & $2.049(0)$ & $2.049(0)$ & $2.049(0)$ \\
& Free & $0(1)$ & $0(1)$ & $0(1)$ & $0(1)$ \\
\hline \multirow{3}{*}{$c=0.2$} & Clamped & $3.993(0)$ & $4.697(0)$ & $5.355(1)$ & $5.714(1)$ \\
& Sim-Supp & $2.135(0)$ & $3.049(0)$ & $3.789(1)$ & $4.039(1)$ \\
& Free & $0.080(1)$ & $0.315(1)$ & $0.662(1)$ & $1.013(1)$ \\
\hline \multirow{3}{*}{$c=0.5$} & Clamped & $4.058(0)$ & $5.894(1)$ & $7.423(2)$ & $8.556(3)$ \\
& Sim-Supp & $2.431(0)$ & $4.351(1)$ & $5.621(0)$ & $6.221(0)$ \\
& Free & $0.492(1)$ & $1.569(1)$ & $1.990(2)$ & $2.239(2)$ \\
\hline \multirow{3}{*}{$c=0.8$} & Clamped & $4.104(0)$ & $6.365(1)$ & $8.941(0)$ & $11.653(1)$ \\
& Sim-Supp & $2.619(0)$ & $5.390(1)$ & $7.967(0)$ & $10.126(1)$ \\
& Free & $1.235(1)$ & $2.718(0)$ & $3.542(0)$ & $4.104(0)$ \\
\hline \multirow{3}{*}{$c=1$} & Clamped & $4.015(0)$ & $6.373(1)$ & $8.968(0)$ & $11.768(1)$ \\
& Sim-Supp & $2.637(0)$ & $5.526(1)$ & $8.449(0)$ & $11.313(0)$ \\
& Free & $1.940(1)$ & $3.879(0)$ & $5.937(0)$ & $7.921(0)$ \\
\hline
\end{tabular}

body tilt when there is no constraint. In general for fixed $c$ the buckling modes switches from $n=1$ to $n=2$ to $n=0$ as stiffness is increased. However, there is a sandwiched pocket of $n=1$ when $\gamma>6$.

Table 1 gives some numerical values form our exact solutions, and therefore serve as a check for approximate numerical methods. Note that a table would not reflect the complex mosaic of mode changes depicted in Figs. 1-3.

\section{References}

Galletly, G.D. (1959), "Circular plates on a generalized elastic foundation”, J. Appl. Mech., 26, 297.

Gupta, U.S. and Ansari, A.H. (1998), "Asymmetric vibrations and elastic stability of polar orthotropic circular plates of linearly varying profile", J. Sound Vib., 215, 231-250.

Gupta, U.S., Ansari, A.H. and Sharma, S. (2006), "Buckling and vibration of polar orthotropic circular plate resting on Winkler foundation", J. Sound Vib., 297, 457-476.

Gupta, U.S., Lal, R. and Jain, S.K. (1991), "Buckling and vibrations of polar orthotropic circular plates of linearly varying thickness resting on an elastic foundation", J. Sound Vib., 147, 423-434.

Hetenyi, M. (1948), Beams on Elastic Foundation. Univ. Michigan, Ann Arbor.

Kline, L.V. and Hancock, J.O. (1965), "Buckling of circular plate on elastic foundation", J. Eng. Ind., 87, 323324.

Wang, C.Y. (2005), “On the buckling of a circular plate on an elastic foundation”, J. Appl. Mech., 72, 795-796.

Wang, C.M., Wang, C.Y. and Reddy, J.N. (2005), Exact Solutions for Buckling of Structural Members CRC Press, Boca Raton.

Wolkowisky, J.H. (1969), "Buckling of the circular plate embedded in elastic springs- an application to geophysics", Commun. Pure Appl. Math., 22, 639-667.

Yu, Y.Y. (1957), "Axisymmetrical bending of circular plates under simultaneous action of lateral load, force in the middle plane, and elastic foundation", J. Appl. Mech., 24, 141-143. 